\title{
Effect of dipole-dipole interactions between atoms in an active medium
}

\author{
Vladimir Berezovsky, ${ }^{1,3, *}$ Leonid Men'shikov, ${ }^{1,2}$ Sven Öberg, ${ }^{3}$ and Chris Latham ${ }^{3}$ \\ ${ }^{1}$ Pomorskii State University, Lomonosov Street, 4, Arkhangelsk, 163002, Russia \\ ${ }^{2}$ Russian Research Center "Kurchatov Institute," Kurchatov Square, 1, Moscow, 123182, Russia \\ ${ }^{3}$ Department of Mathematics, Luleå University of Technology, SE-97187 Luleå, Sweden \\ *Corresponding author: vladimir@sm.luth.se
}

Received July 27, 2007; revised January 8, 2008; accepted January 8, 2008; posted January 9, 2008 (Doc. ID 85783); published February 29, 2008

\begin{abstract}
On the basis of the results of numerical modeling, it is shown that dipole-dipole interactions among atoms in the active medium strongly influences the character of the associated superradiation. The main effect is to make the nuclear subsystem behave chaotically. Its strength increases with the atom density and leads to the suppression of distant collective correlations and superradiation. Near correlations between the atoms are established, causing a confinement effect: a shielding of radiation in the active medium. (C) 2008 Optical Society of America
\end{abstract}

OCIS codes: $020.1670,140.6630,270.6630$.

\section{INTRODUCTION AND METHOD}

Superradiation (SR) is the cooperative radiation arising in a medium that contains a population inversion of excited states. Originally, this effect was stated for purely quantum systems, i.e., two-level atoms [1]. Experiments have confirmed this prediction [2]. Later work established that this phenomenon also occurs in classical systems $[3,4]$, and that the phasing effect- the spontaneous origin and strengthening of correlations of originally independent subsystems-underlies it. In the quantum case, these are correlations among phases of electronic states of atoms undergoing radiative transitions, whereas in the classical regime correlations among phases of oscillations and directions of the electric-dipole moments of atoms occur. A full account of the influence on SR of the dipoledipole interactions among atoms remains incomplete (see [5-7]).

The SR theory has been developed from several directions. There exist complementary to each other Schrödinger, Heisenberg, and semiclassical approaches. Each approach is applicable to a special area of values of the system parameters. The common methodological lack of these approaches is that the phasing mechanism remains off screen. The mechanism of the transition from casual to a phased state possesses certain spatial, time, and statistical behaviors, and its nature is not fully clear. The quantum-mechanical problem of SR is rather complicated; for example, within the Heisenberg approach it requires to solve a system of nonlinear operational equations. Approximations that are used to simplify this system have a limited and often unclear area of applicability. The classical model of superradiation (CMS), where atoms are substituted by the classical Lorenz oscillators and the electromagnetic field is described by the classical Maxwell equations, allows us to answer many difficult questions; in particular, the phasing mechanism. Therefore classical and quantum approaches complement each other. Moreover, radiation produced by pure classical system such as electrons revolved in magnetic field, electron clouds created in wigglers, cathode-ray lamps for microwaves, etc., is also SR.

Let us consider only classical systems. First, phasing leads to the ordering of the phases of atoms. Second, according to Earnshaw's theorem [8,9], a system of point dipoles cannot maintain a stable static equilibrium configuration. Dipole-dipole interactions cause chaotic behavior that disorders their phases and hence suppresses SR. SR arises from a competition between these two opposing effects. This assumption was made in $[7,10,11]$. The aim of this paper is to verify this assumption by computer simulations.

Consider now a nonlinear CMS [7,12], i.e., a system of classical, charged anharmonic oscillators. Anharmonicity means that the vibrational frequency of the oscillator depends on its energy: $\omega=\omega(E)$. According to Gaponov [3,4] (see also a simplified explanation [13]) such a dependence is a key physical reason of phasing. Maxwell's equations describe the electromagnetic field in CMS. Next, assume that there are sufficient oscillators $(N \gg 1)$, and they occupy a spatial region of length $L$ such that $l \ll L$, where $l$ $=n^{1 / 3}$ is the characteristic distance between atoms. Each charge has magnitude $e$ and mass $m$ and is located on the ends of springs with stiffness coefficient $k$, at coordinates $\mathbf{r}_{a}+\xi_{a}(a=1,2, \ldots, N)$, fixed in points $\mathbf{r}_{a}$, where there are also compensating charges $-e$. The equation of motion for the oscillators then takes the form ([14]; see also Appen$\operatorname{dix} \mathrm{A})$

$$
\ddot{\boldsymbol{\xi}}_{a}+\omega_{0}^{2}\left(1+\gamma \xi_{a}^{2}\right) \xi_{a}+\frac{2 e^{2} \omega_{0}^{2}}{3 m c^{3}} \dot{\boldsymbol{\xi}}_{a}=\frac{e^{2}}{m} \sum_{b \neq a} \nabla_{a} \times\left(\nabla_{a} \times \frac{\xi_{b}\left(t_{a b}\right)}{r_{a b}}\right) .
$$

Here $\nabla_{a}=\partial / \partial \mathbf{r}_{a}, \mathbf{r}_{a b}=\mathbf{r}_{a}-\mathbf{r}_{b}, t_{a b}=t-r_{a b} / c$ represents the retarded time, $\omega_{0}=\sqrt{\mathrm{k} / \mathrm{m}}$ is the fundamental frequency of 
the oscillators, and $\gamma$ is the nonlinearity parameter. Substituting the expression

$$
\boldsymbol{\xi}_{a}=b\left[\mathbf{F}_{a}(t) \exp (-\iota \omega t)+\mathbf{F}_{a}^{*}(t) \exp (\iota \omega t)\right],
$$

into Eq. (1)—where $b$ represents the characteristic initial amplitude of the oscillations-gives

$$
\begin{aligned}
\dot{\mathbf{F}}_{a}+\iota \delta\left(\left|\mathbf{F}_{a}\right|^{2}-1\right) \mathbf{F}_{a}+\frac{1}{2} \beta_{0} \mathbf{F}_{a}= & \iota \beta \sum_{b \neq a} \nabla_{a} \times\left[\nabla_{a} \frac{\exp \left(\iota \mathbf{k} \mathbf{r}_{a b}\right)}{r_{a b}}\right. \\
& \left.\times \mathbf{F}_{b}(t)\right]
\end{aligned}
$$

In Eq. (3) the second derivatives of functions $F_{a}(t)$, which vary slowly in comparison with exponents $\exp ( \pm \imath \omega t)$, are omitted, and a frequency $\omega=\omega_{0}+\delta, \quad \delta$ $=3 \gamma \omega_{0} b^{2} / 2$ is chosen. Note that, in the case of particles rotating in a magnetic field $\mathbf{B}$ (important in a practical sense), the rotation frequency $\omega_{H}$ is equal to

$$
\frac{e B}{m c} \sqrt{1-\frac{v^{2}}{c^{2}}} \approx \frac{e B}{m c}\left(1-\frac{v^{2}}{2 c^{2}}\right)[14] \text {. }
$$

This means that $\mathrm{d} \omega_{H} / \mathrm{d} E<0$ corresponds to $\delta<0$.

For a small-size system $L \ll \lambda=2 \pi c / \omega_{0}$ the Taylor series expansion from Appendix A should be applied to every term of the right-hand side of Eq. (3), giving the total radiative friction electric field $\mathbf{E}_{r}=2 / 3 c^{3} \mathbf{D}, \mathbf{D}=e \sum_{a=1}^{N} \boldsymbol{\xi}_{a}$ [14]. After neglecting the retardation effects one obtains the following system:

$$
\dot{\mathbf{F}}_{a}+\iota \delta\left(\left|\mathbf{F}_{a}\right|^{2}-1\right) \mathbf{F}_{a}=\iota \beta \sum_{b \neq a} \frac{3 \mathbf{n}_{a b}\left(\mathbf{n}_{a b} \mathbf{F}_{b}\right)-\mathbf{F}_{b}}{r_{a b}^{3}}-\frac{1}{2} \beta_{0} \sum_{b} \mathbf{F}_{b}
$$

where $\mathbf{n}_{a b}=\mathbf{r}_{a b} / r_{a b}, \quad \beta=e^{2} /\left(2 m \omega_{0}\right)$, and $\beta_{0}=2 e^{2} \omega_{0}^{2} / 3 m c^{3}$. The first term on the right-hand side of Eq. (4) represents the dipole-dipole interaction of the oscillators, whereas the second term is analogous to a "viscosity" for the radiation in the electromagnetic field. Following [12], we shall consider one-dimensional oscillators, i.e., dipoles that oscillate along the $\boldsymbol{x}$ axis, and consequently, that the vectors $\mathbf{F}_{a}$ are parallel to it: $\mathbf{F}_{a}=\mathbf{i} F_{a}, \mathbf{i}=(1,0,0)$. During a given time $t$ we have $F_{a}(t)=\rho_{a}(t) \exp \left[\imath \varphi_{a}(t)\right]$. Hence, atoms possess a dipole moment that is $\mathbf{d}_{a}(t)=e \boldsymbol{\xi}_{a}=e b \mathbf{i} \rho_{a} \cos \left(\omega t+\varphi_{a}\right)$.

The average radiation intensity of the rapidly oscillating dipoles then is

$$
I=\frac{e^{2} \omega^{4} b^{2}}{3 c^{3}} \sum_{a, b}\left|F_{a}\right|\left|F_{b}\right| \cos \left(\varphi_{a}-\varphi_{b}\right)
$$

Thus, Eq. (4) represents a system of $N$ oscillators, distributed arbitrarily, that can be solved by numerical means. A similar formalism is described in [12]; however, dipole-dipole interactions are neglected.

\section{RESULTS AND DISCUSSION}

The phasing effect can be described as follows. Consider a complex plane $(x, y)=[\mathfrak{R}(F), \Im(F)]$ containing $N$ points that each represent the state of an individual oscillator, where the distance from the origin is simply the ampli-

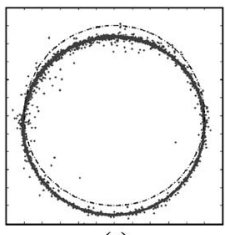

(a)

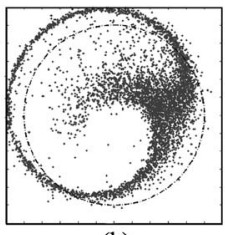

(b)

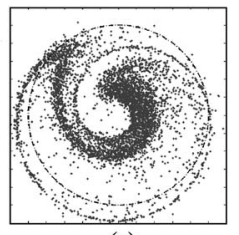

(c)
Fig. 1. Time evolution of the phase distribution of oscillators. The dotted curve is a circle with unit radius. The number of oscillators is $N=5 \times 10^{3}$. The concentration of oscillators $n$ $=10^{22} \mathrm{~m}^{-3}$ (curve 2 in Fig. 4). The $x$ and $y$ axes correspond to $x_{a}$ $=\rho_{a} \cos \varphi_{a}, y_{a}=\rho_{a} \sin \varphi_{a}$, where $\rho_{a}$ and $\varphi_{a}$ were introduced above Eq. (5).

tude of oscillation and the angle is the phase with respect to the fundamental frequency $\omega_{0}$. Points with $\omega>\omega_{0}$ rotate clockwise around the origin; points with $\omega<\omega_{0}$ rotate counterclockwise.

Initially, the points are placed randomly with equal probability phases on a circle of unit radius $\rho=1$. From Eq. (4), their velocities are

$$
\mathbf{v}_{a}=\boldsymbol{\omega}\left(\rho_{a}\right) \times \boldsymbol{\rho}_{a}+\mathbf{f}+\sum_{b} \mathbf{d}\left(\rho_{a}, \rho_{b} ; \mathbf{r}_{a}, \mathbf{r}_{b}\right)
$$

Here the following notations are introduced: $\rho_{a}$ $=\left[\Re\left(F_{a}\right), \Im\left(F_{a}\right), 0\right], \mathbf{v}_{a}=\boldsymbol{\rho}_{a}, \mathbf{f}=-\beta_{0} \Sigma_{a} \boldsymbol{\rho}_{a} / 2$, and $\boldsymbol{\omega}(\rho)=[0,0$, $\left.-\delta\left(\rho^{2}-1\right)\right], \mathbf{d}\left(\rho_{a}, \rho_{b} ; \mathbf{r}_{a}, \mathbf{r}_{b}\right)$. The latter dipole-dipole interaction term is not shown in full for reasons of space. Note that the vector $-\mathbf{f}$ is proportional to the total dipole moment of the system $\mathbf{D}=e b \Sigma_{a} \boldsymbol{\rho}_{a} / 2$, and $\omega\left(\rho_{a}\right)=0$ at $t=0$.

Notice also that the sign of $\gamma$ affects only the direction of rotation; changing it results in a mirror inversion without any other consequences. Points with positive $\gamma$ rotate clockwise outside the unit circle and rotate counterclockwise when inside, whereas the opposite is true when $\gamma$ is negative. This symmetry, therefore, is exploited by choosing $\gamma>0$.

Having established the basis for the model, we next consider how the system evolves when the density of atoms $n$ is sufficiently small that dipole-dipole interactions are negligible. During to the fluctuations of density distribution of the oscillators initial phases $\varphi_{a}(0)$, the initial value of the vector $\mathbf{f}$ is not precisely zero. At $t=0$ from Eq. (6) it follows that $\mathrm{d} \mathbf{D} / \mathrm{d} t=-\mathbf{D} / \tau_{S R}$, where the characteristic emission time is $\tau_{S R}=1 /\left(N \beta_{0}\right)$ [1,5-7].

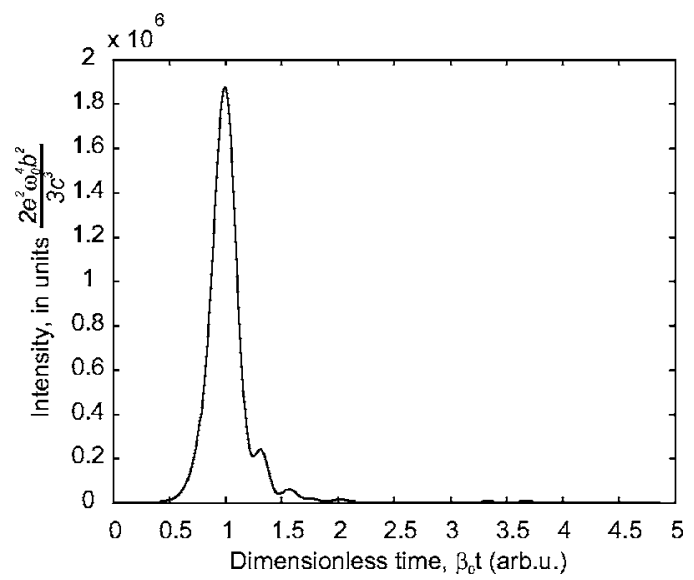

Fig. 2. Time dependence of the radiation intensity for $N=5$ $\times 10^{3}$ (all values in arbitrary units). 


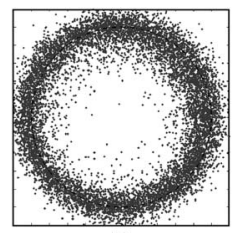

(a)

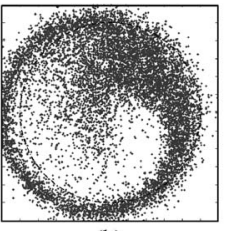

(b)

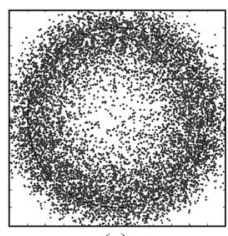

(c)
Fig. 3. Time evolution of the phase distribution of oscillators in systems with a strong dipole-dipole interaction. The dotted curve is a circle with unit radius. (a) and (b) correspond to the concentration of oscillators $n=8 \times 10^{22} \mathrm{~m}^{-3}$ (curve 4 in Fig. 4). (c) corresponds to $n=1.8 \times 10^{23} \mathrm{~m}^{-3}$ (curve 6 in Fig. 4). Notations are the same as in Fig. 1.

Consequently from Eq. (6), the system responds by moving in a direction opposite to the dipole moment $\mathbf{D}$, with a collective net velocity $\mathbf{f}$. The system at time $\sim \tau_{S R}$ is displaced a distance $\sim \mathbf{D}(0) /(N e)$ [see Fig. 1(a)]. The resulting displacement moves half of the points outside the unit circle $(\rho>1)$, and the other half inside $(\rho<1)$. Hence, points outside the circle will move in clockwise orbits, while those within circulate the opposite way. After an interval $t \sim 10 \tau_{S R}$, the net motion results in a bunching of points on the inside of the circle [Fig. 1(b)], thus the atoms emit most of their stored energy in a sharp pulse of coherent radiation (Fig. 2). For two-level atoms, the characteristic delay time $t_{0}=\tau_{S R} \log N$ given in [1] is consistent with this. The bunch subsequently develops into a spiralshaped distribution [Fig. 1(c)]. As it does so, the dipole moment decreases to a minimum, along with the SR intensity. The cycle repeats, decaying rapidly (Fig. 2). Oscillatory behavior is typical for SR in classical systems of small size [12]. In quantum systems consisting of twolevel atoms, SR intensity oscillations are absent [1].

At high density $n$, dipole-dipole interactions have a significant effect. Figure 3 shows the outcome of Eq. (4) for large $n$; the initial conditions are the same as described previously. Notice that the points on the phase plane now move in a more chaotic manner than before. When $n$ is high, dipole-dipole interactions among adjacent oscillators are strong, and this leads to incoherence. However, $\mathrm{SR}$ is not entirely suppressed. In spite of the chaotic behavior of dipole-dipole interaction, the initial total dipole

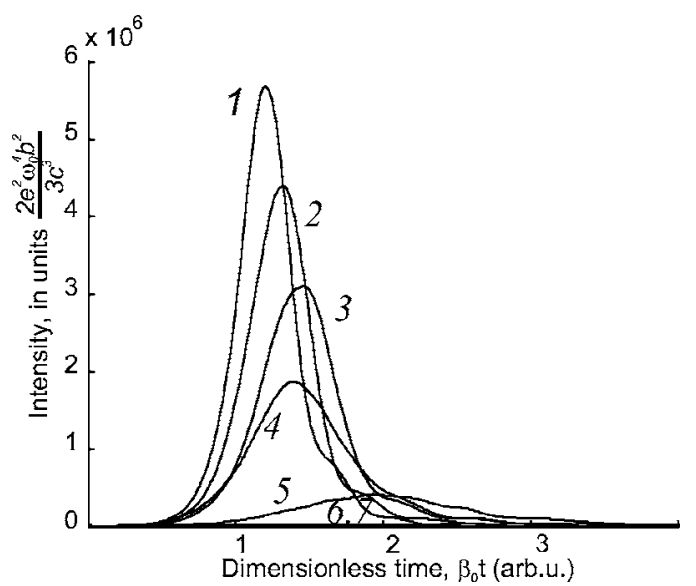

Fig. 4. Intensity of radiation (arbitrary units) for systems with different oscillator concentrations $n\left(10^{22} \mathrm{~m}^{-3}\right)$ : $0.083,1.0,2.3$, $8.0,12.13,18.38$, and 27.86 for curves $1-7$, respectively. Units coincide with those of Fig. 2.

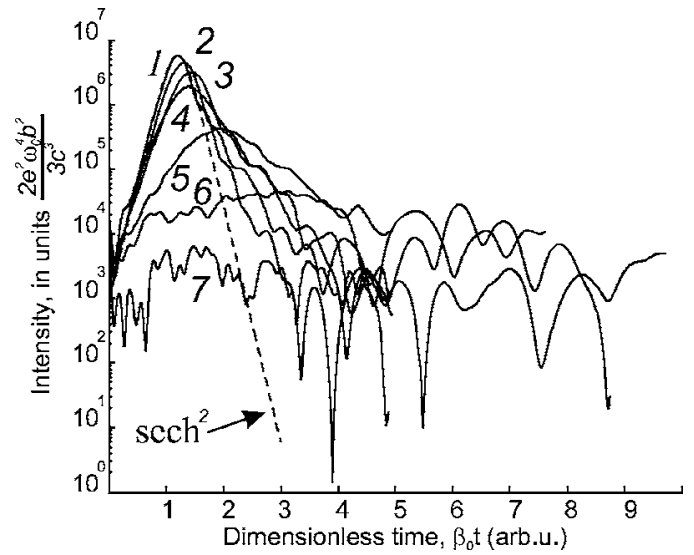

Fig. 5. Radiation intensity (arbitrary units) versus time (arbitrary units) for classical systems with different oscillator concentrations $n\left(10^{22} \mathrm{~m}^{-3}\right): 0.083,1.0,2.3,8.0,12.13,18.38$, and 27.86 for curves $1-7$, respectively. Case 1 is compared with the purely quantum result, which varies as $\operatorname{sech}^{2}\left(t-t_{0}\right)$. Units coincide with those of Fig. 2.

moment results in bunching of points, and correspondingly in the SR pulse [Figs. 3(a) and 3(b)]. In Fig. 3(c), where the concentration of oscillators was doubled, dipole-dipole interaction suppress the bunching.

High-density systems are also complicated by collective effects. Localized groups of resonant atoms induce antiphase dipole moments among their neighbors. This preserves coherence while screening SR [7].

The SR delay $t_{0}$ and peak intensity $I_{\max }$ also depend on $n$; increasing $n$ makes $t_{0}$ longer and $I_{\text {max }}$ smaller (see Figs. 4-6). This is a consequence of the effect of coherence on the collective interactions among the dipoles, which becomes weaker with increasing $n$.

Unlike classical systems, quantum systems do not behave chaotically. The intensity varies smoothly with time as described by the following formula [1]:

$$
I(t)=\frac{\hbar \omega_{0}}{4 \mu \tau_{N}}(\mu N+1)^{2} \operatorname{sech}^{2}\left(\frac{t-t_{0}}{2 \tau_{N}}\right),
$$

where $\mu$ represents the form factor of the oscillators' mutual position and $\tau_{N}=1 / \beta_{0}$ is the characteristic emission time. This curve is plotted in Fig. 5 to illustrate the dif-

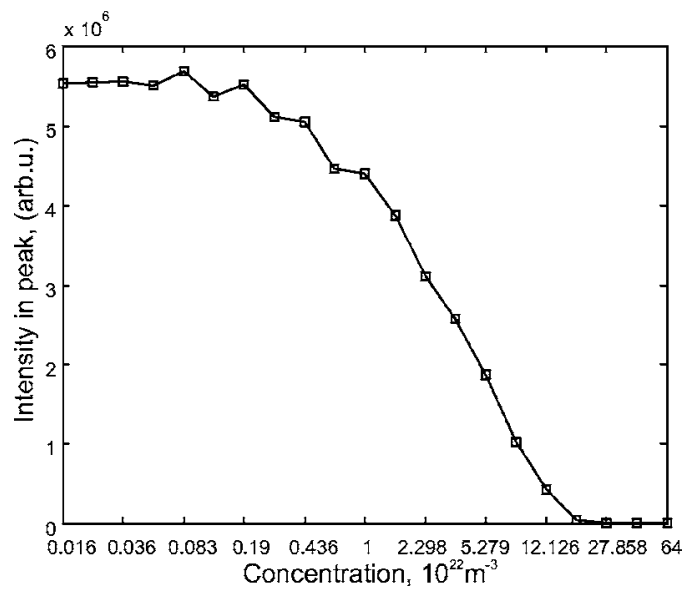

Fig. 6. Dependence of a maximum of radiation intensity (arbitrary units) on oscillator density $n$ (in $10^{22} \mathrm{~m}^{-3}$ ). 


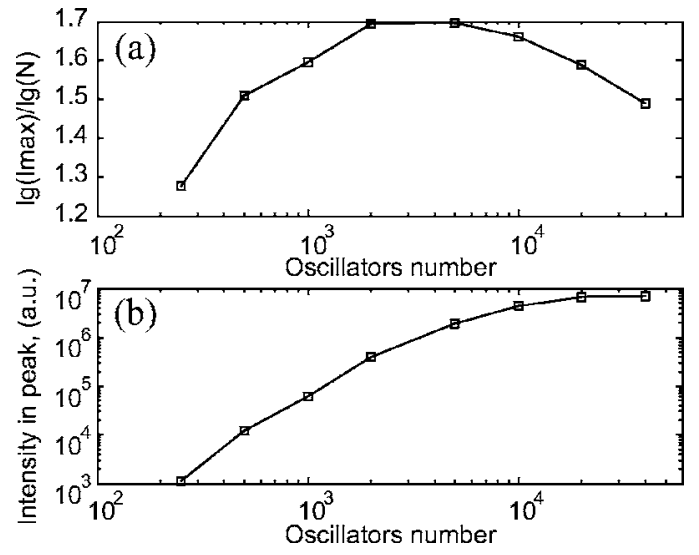

Fig. 7. Dependencies on the number of oscillators $N$, of (a) the ratio $\log _{10}\left(I_{\text {max }}\right) / \log _{10}(N)$, and (b) the peak of radiation intensity $\left(I_{\max }\right)$ (the latter is in arbitrary units).

ference between the classical and quantum cases. When $N$ is large, at $t=t_{0}$, Eq. (7) suggests $I_{\text {max }} \sim N^{2}$. However, the CMS predicts that the exponent $\alpha=\lg \left(I_{\text {max }}\right) / \lg (N)$ rises to a peak value that is less than two then declines as $N$ increases (see Fig. 7). Experimental observations of SR in semiconductors show that similar behavior is exhibited [15].

These results are consistent with [7]. Localized, dynamic metastable states are formed when the atom density $n$ is sufficiently large. Each oscillator perturbs the motion of its nearest neighbors such that their relative phase differs by $\pi$. Hence, in effect each oscillator appears to be screened in a manner analogous to Debye shielding. This leads to confinement of electromagnetic fields in the active medium. This effect holds true for the systems of all dimensions, both small $(L<\lambda)$ and extended $(L>\lambda)$.

\section{CONCLUSIONS}

This study examines the phenomenon of SR for systems of classical nonlinear charged oscillators. The results of our numerical simulations show that after a characteristic delay time $t_{0}$, a peak in radiated power occurs that subsequently decays in a chaotic, oscillatory manner, superimposed on a $\operatorname{sech}^{2}\left(t-t_{0}\right)$ background. SR is also suppressed progressively with increasing oscillator density $n$. This behavior is ultimately a consequence of collective dipoledipole interactions. These both induce incoherence among the oscillators and cause a screening effect.

Within localized regions, the individual dipoles possess correlated moments. Dipoles separated by sufficient large distances are nearly uncorrelated. As $n$ increases, the system breaks up into more of these regions. Each region emits SR impulses independently, resulting in the chaotic decay described above.

Some notes should be added in connection with the relation between the quantum two-level atoms model [1] and CMS. As far as we know no one has investigated this question in detail. It is clear that there is no quantitative analogy between these two opposite cases. But we believe that the qualitative one really exists. The essence of this analogy is the phasing effect in CMS that is similar to the quantum coherence that arose between initially independent atoms in [1].

\section{APPENDIX A: DERIVATION OF EQ. (1)}

Let us suppose that at the points with coordinates $\mathbf{r}_{a}(a$ $=1, \ldots, N)$ springs are fixed and the compensating charges $(-e)$ are placed in each of them. The point masses $m$ having charges $(+e)$ are fixed on the ends of the springs. Coordinate of this charge with respect to the point $\mathbf{r}_{a}$ is $\xi_{a}$. Potential energy of the springs is given by

$$
U\left(\xi_{a}\right)=\frac{1}{2} m \omega_{0}^{2} \xi_{a}^{2}+\frac{1}{4} \gamma m \omega_{0}^{2} \xi_{0}^{4} .
$$

For nonrelativistic motion $\left|\dot{\xi}_{a}\right| \ll c$ it is possible to neglect the influence of the magnetic field, henceforth the equations of motion take the form

$$
m \ddot{\boldsymbol{\xi}}_{a}+m \omega_{0}^{2}\left(1+\gamma \xi_{0}^{2}\right) \boldsymbol{\xi}_{a}=e \mathbf{E}\left(r_{a}, t\right)
$$

Here

$$
\mathbf{E}(\mathbf{r}, t)=e \sum_{b=1}^{N} \nabla_{r} \times\left[\nabla_{r} \times \frac{\boldsymbol{\xi}_{b}\left(t-\frac{R_{b}}{c}\right)}{\mathbf{R}_{b}}\right]
$$

is the electric field of pointlike electric dipole moment $\mathbf{d}_{b}(t)=e \boldsymbol{\xi}_{b}(t)$ at the point with coordinate $\mathbf{r}, \mathbf{R}_{b}=\mathbf{r}-\mathbf{r}_{b}[14]$. After insertion of (A3) into (A2) the infinite term with $b$ $=a$ will arise. To correct calculation of this term it is necessary to smear point charges $(+e)$ and $(-e)$ over a small finite region. Then one should produce the Taylor series expansion of function $\xi_{a}\left[t-\left(R_{a} / c\right)\right]$ with respect to $1 / c$ up to the third-order terms $\left[\sim\left(1 / c^{3}\right)\right]$. The term $\sim 1$ gives the self-electric field of each charge. Of course, that field can not accelerate this charge, therefore the correspondent terms $\sim 1$ should be omitted. The field of charge $(-e)$ also produces the force, acting on the charge $(+e)$ located at another end of the common spring. This force should be included into the potential energy of the spring [Eq. (A1)]. The stiffness coefficient $k$ renormalization allows us to omit this term. In other words, one should consider the potential energy [Eq. (A1)] as a resulting energy of the spring. Next, the term $\sim(1 / c)$ vanishes, as it is clear from Eq. (A3). The term $\sim\left(1 / c^{2}\right)$ is $-(4 / 3) m_{f} \ddot{\xi}_{a}$, where $m_{f}$ $=\left(\epsilon_{f} / c^{2}\right)$ and $\epsilon_{f}$ are the mass and the energy of the electromagnetic cloud surrounding a spring, correspondingly. This term should be included into the mass $m$ (the mass renormalization). The strange factor $4 / 3$ represents a well-known paradox "4/3" (see [16], for example). Finally, the term $\sim\left(1 / c^{3}\right)$ equals $\mathbf{F}_{a}^{(r)}=\left(2 e^{2} / 3 c^{3}\right) \ddot{\boldsymbol{\xi}}_{a}$. This term corresponds to the radiational friction, which is responsible for the radiational damping of a dipole moment of an isolated atom. Further, it is necessary to take the approximation $\ddot{\boldsymbol{\xi}}_{a} \approx-\omega_{0}^{2} \boldsymbol{\xi}_{a}$, giving $\mathbf{F}_{a}^{(r)} \approx\left[\left(2 e^{2} \omega_{0}^{2}\right) /\left(3 c^{3}\right)\right] \boldsymbol{\xi}_{a}$. This approximation gives the possibility to avoid incorrect regimes of charge "self-acceleration" resulting from omitting the term $\sim\left(1 / c^{4}\right)$ [14]. Finally, the equations of motion take the form of Eq. (1), leading to Eq. (3). These equations hold for the nonrelativistic system of nonlinear oscillators of any shape and dimension. 


\section{ACKNOWLEDGMENTS}

This work was supported by the Swedish Institute, Luleå University of Technology and by a grant from the administration of Arkhangelsk region, Russia, 2007, project 03-3.

\section{REFERENCES}

1. R. H. Dicke, "Coherence in spontaneous radiation processes," Phys. Rev. 93, 99-110 (1954).

2. N. Skribanowitz, I. P. Herman, J. C. MacGillivray, and M. S. Feld, "Observation of Dicke superradiance in optically pumped HF gas," Phys. Rev. Lett. 30, 309-312 (1973).

3. A. V. Gaponov, "Instability of a system of excited oscillators with respect to electromagnetic perturbations," Sov. Phys. JETP 12, 232-298 (1960).

4. A. V. Gaponov, M. I. Petelin, and V. K. Yulpatov, "The induced radiation of excited classical oscillators and its use in high-frequency electronics," Radiophys. Quantum Electron. 10, 794-823 (1967)

5. M. Gross and S. Haroche, "Superradiance: an essay on the theory of collective spontaneous emission," Phys. Rep. 93, 301-396 (1982).

6. S. Stenholm, "Quantum theory of electromagnetic fields interacting with atoms and molecules," Phys. Rep. 6, 1-121 (1973).

7. L. I. Men'shikov, "Superradiance and related phenomena," Sov. Phys. Usp. 42, 107-147 (1999).

8. D. V. Sivukhin, General Course of Physics: Vol. 3: Electricity (Nauka-Fizmatlit, 1996).

9. J. A. Stratton, Electromagnetic Theory (McGraw-Hill, 1941).

10. R. Friedberg, S. R. Hartmann, and J. T. Manassah, "Limited superradiant damping of small samples," Phys. Lett. A 40, 365-366 (1972).

11. R. Friedberg and S. R. Hartmann, "Temporal evolution of superradiance in a small sphere," Phys. Rev. A 10, 1728-1739 (1974).

12. Yu. A. Il'inskii and N. S. Maslova, "The classical analog of superradiation in a system of interacting nonlinear oscillators," Sov. Phys. JETP 94, 171-174 (1988).

13. V. V. Berezovskii and L. I. Men'shikov, "Transverse cooling of electron beams," JETP Lett. 86, 355-357 (2007).

14. L. D. Landau and E. M. Lifshiz, Course of Theoretical Physics: Vol. 2: the Classical Theory of Fields (Pergamon, 1975).

15. S. V. Zaitsev, L. A. Graham, D. L. Huffaker, N. Yu. Gordeev, V. I. Kopchatov, L. Ya. Karachinsky, I. I. Novikov, and P. S. Kop'ev, "Superradiance in semiconductors," Sov. Phys. Semicond. 33, 1309-1314 (1999).

16. W. Pauli, Theory of Relativity (Dover, 1981) 